\title{
SURVEY OF LOCAL BINARY PATTERN FOR FIRE \& SMOKE USING WAVELET DECOMPOSITION
}

\author{
Akshata Patil ${ }^{1}$, Varsha Bhosale ${ }^{2}$ \\ ${ }^{I}$ Student, Vidyalankar Institute of Technology, Wadala, Mumbai \\ ${ }^{2}$ Lecturer, Vidyalankar Institute of Technology, Wadala, Mumbai
}

\begin{abstract}
Current Automatic Smoke Detection Mechanism's, i.e. Fire Alarms, include sensor technologies, which are unreliable, as the alarm can go off, even though there is no fire or smoke. Using image processing a quite accurate system for smoke detection and amount of smoke generated, i.e. seriousness of the condition when there is a fire can be estimated. So, a pixel level analysis is required.
\end{abstract}

Keywords: Smoke Detection, Image Segmentation, Color Space Model, Fuzzy Inference System.

\section{INTRODUCTION}

For fire alert system, the most important problem is how quick the existence of fire is detected. The fire detection in open areas, image information is mainly used due to the fact that other sensing devices such as a gas sensor become high cost and cannot set them in some places. Some methods of detecting a fire or flame directly are proposed, however, they have some difficulties to catch fire with cameras depending on the relation of places between a camera position and a fire generating place. For such purpose, smoke is a key feature since we can observe it even if fire is not in the field of view of the camera. There are some computer vision based methods for smoke detection which use the edge or color information based technique. They have difficulties in treating characteristic properties of smoke and needing high-cost computations to detect smoke whole of wide-view images or image sequences. There are other approaches which combine texture features and its time-series properties. It is shown that they can provide accurate results of smoke detection in open areas. However, they need relatively long time to obtain the result as they use the time accumulation technique, which becomes the problem in some real world situations. Additionally, these methods have a common problem, which is that the detection result is affected from an illumination change. This becomes a serious problem for open area image processing as whether conditions, for examples, clouds, lighting sources, wind, etc., are always changing dynamically.

\section{EXISTING METHOD}

In one of the existing method, fuzzy output is used for better discrimination of fire and fire-like coloured objects with respect to defined rules. In this method, they have supported with fuzzy logic \& defined $\mathrm{YCbCr}$ color space model.
For fire detection, they have considered characteristics of color channels $\mathrm{Y}, \mathrm{Cb} \& \mathrm{Cr}$. Fire pixel is having $\mathrm{Y}(\mathrm{x}, \mathrm{y})$ greater than $\mathrm{Cb}(\mathrm{x}, \mathrm{y})$ luminance information related to the intensity expected dominant to the fire pixel. Greater difference between $\mathrm{Y}(\mathrm{x}, \mathrm{y})$ \& $\mathrm{Cb}(\mathrm{x}, \mathrm{y})$, is then also considered as a fire pixel. $\mathrm{Cb}(\mathrm{x}, \mathrm{y})$ should be smaller than $\mathrm{Cr}(\mathrm{x}, \mathrm{y})$. Higher difference between $\mathrm{Cb}(\mathrm{x}, \mathrm{y}) \& \mathrm{Cr}(\mathrm{x}, \mathrm{y})$, then it is fire pixel.

For smoke detection, Smoke pixel do not show chrominance characteristics. Temperature of smoke is low, smoke will show color from range of white-blurish to white. Start of fire, smoke temperature increases gets from blackgreyish to black. Smoke sample is detailed when smoke has low temperature. For these purpose they have used HSV color space model to defined saturation of color should be low as possible. According to the reflectance property, smoke pixel around fire pixel also defined as fire.

Table 1.1 Rule table for fuzzy inference system.

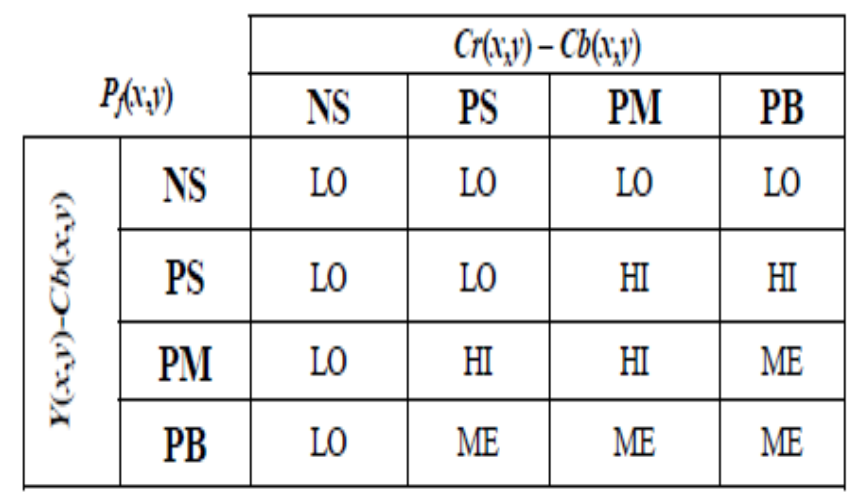




\subsection{Material \& Methodology}

1. Take input image

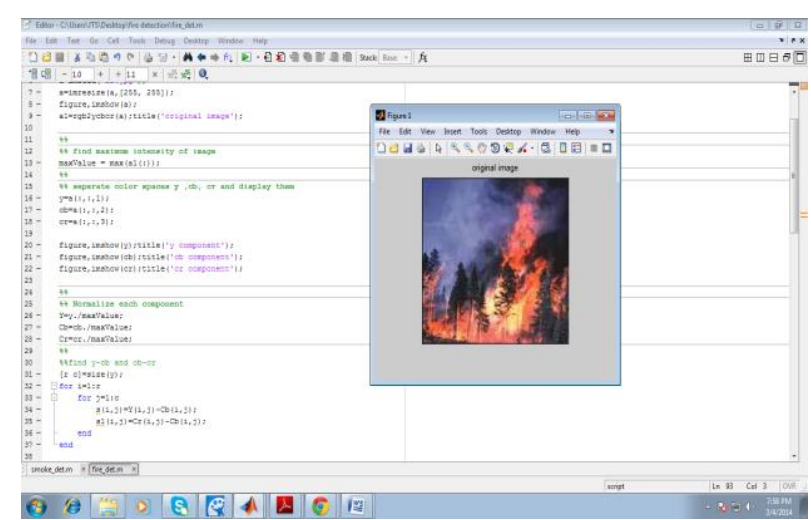

Fig. 1.1 Input Image

2. Convert into ycbcr color space.

3. Display each color space.

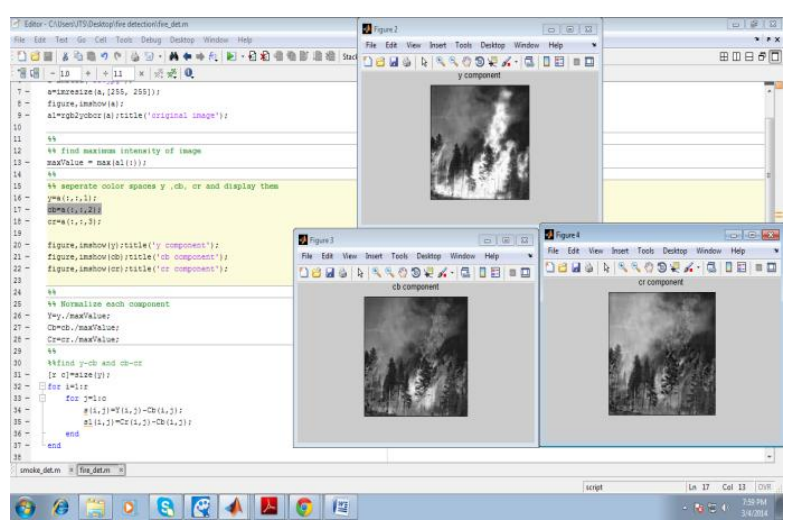

Fig 1.2 YCbCr color space model

4. Normalize each color space component.

5. Find $y-c b$ and cb-cr

6. Initialize fis structure.

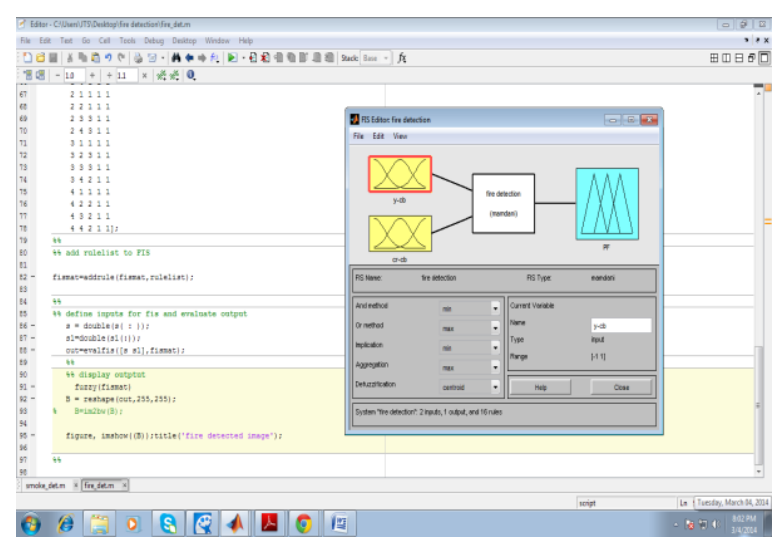

Fig.1.3. Fuzzy Inference System

7. Add rules to fis structure.

8. Convert $\mathrm{y}-\mathrm{cb}$ and $\mathrm{cr}-\mathrm{cb}$ into row vector

9. Evaluate fis structure

10. Reshape output to $255 * 255$

11. Display output viz. detected fire in image.

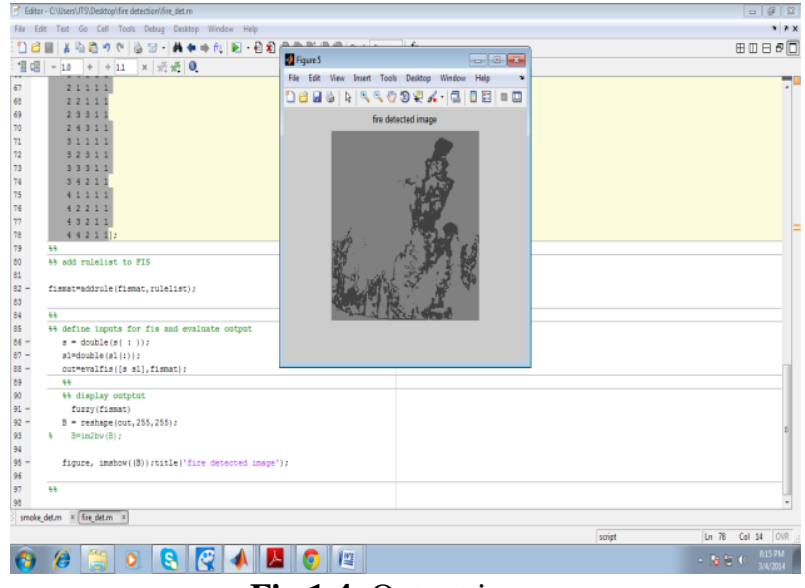

Fig.1.4. Output image

\section{Smoke Detection:}

1. In smoke detection input image is converted into hsv color space.

2. Display each color space.

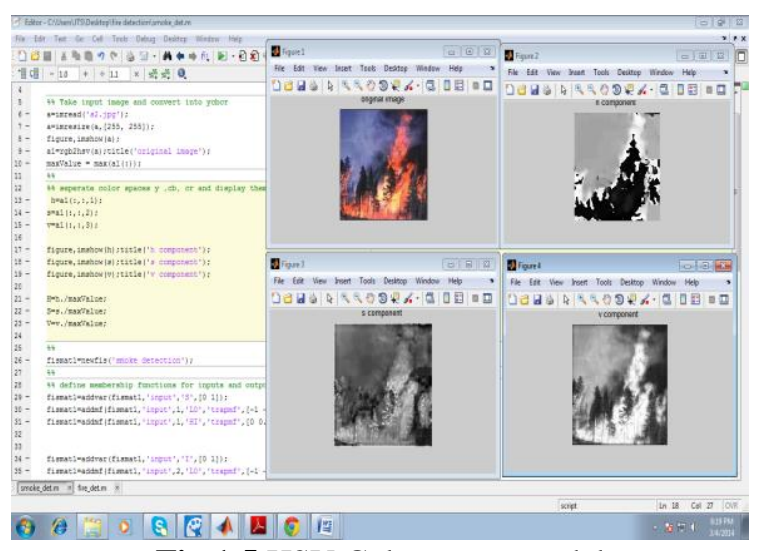

Fig.1.5 HSV Color space model

3. Convert $\mathrm{s}$ and v color space into row vector

4. For fuzzy part follow same procedure of smoke detection.

5. Evaluate output and reshape into $255 * 255$

6. Convert output into logical and display.

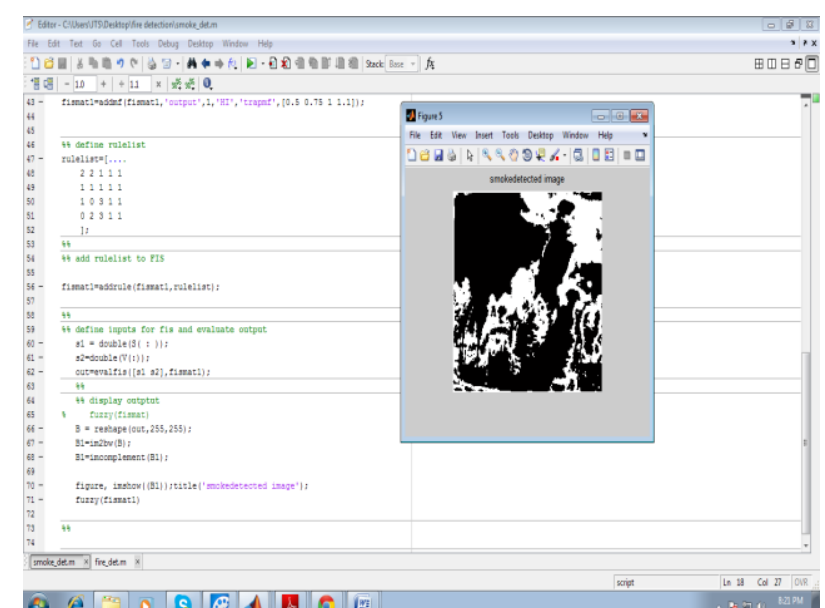

Fig 1.6 Output Image 


\section{PROPOSED METHOD}

Current Automatic Smoke Detection Mechanism's, i.e. Fire Alarms, include sensor technologies, which are unreliable, as the alarm can go off, even though there is no fire or smoke. Using Image Processing a quite accurate system for smoke detection and amount of smoke generated, i.e. seriousness of the condition when there is a fire can be estimated. So, a pixel level analysis is required.

In this study, we adopt a simple texture feature named Local Binary Patterns (LBP). LBP is defined as a simple texture operator computed using the center pixel value and its neighborhood pixel values. As it is considering the result as a binary number, LBP is robust against the illumination change. Therefore, it can be suitable for our purpose of this study. Additionally, we also adopt wavelet method to improve the accuracy of detection results. When we prepare a training set, we need to consider that the image information of smoke is strongly affected from its background. That is, we have difficulties to gather smoke images and non-smoke images in a wide range of variations. Wavelet is one of the widely used learning method to classify the complicated data in real world. We consider smoke detection as a two-class problem in the context of learning problem. In such situation, we adopt wavelet to our method to improve the accuracy as it is suitable our purpose.

A method for smoke detection in video is proposed. It is assumed the camera monitoring / satellites captured imaged, the scene is stationary. Since the smoke is semitransparent, edges of image frames start losing their sharpness and this leads to a decrease in the high frequency content of the image. To determine the smoke in the field of view of the camera, the background of the scene is estimated and decrease of high frequency energy of the scene is monitored using the spatial wavelet transforms of the current and the background images. Edges of the scene are especially important because they produce local extrema in the wavelet domain. A decrease in values of local extremais also an indicator of smoke. In addition, scene becomes grayish when there is smoke and this leads to a decrease in chrominance values of pixels. Periodic behavior in smoke boundaries and convexity of smoke regions are also analyzed. All of these clues are combined to reach a final decision.

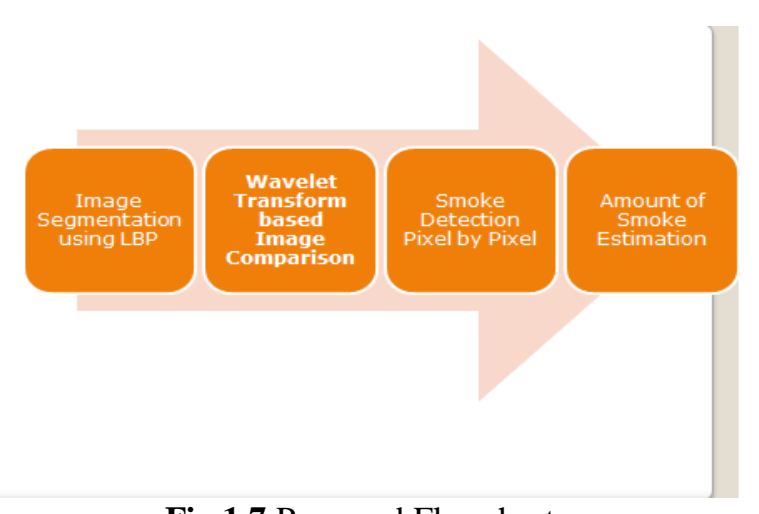

Fig 1.7 Proposed Flowchart

\section{Local Binary Pattern}

Local binary patterns of LBP deals with the neighbor pixels in an image by using thresholding the neighbourhood of each pixel and consider the result as a binary number, and thus, is used for segmentation and clustering in an image.

\section{Wavelet Transform}

In mathematics, a wavelet series is a representation of a square integrable (real- or complex valued) function by a certain orthonormal series generated by a wavelet. Nowadays, wavelet transformation is one of the most popular candidates of the time-frequency transformations.

\subsection{Material \& Methodology}

\subsubsection{Preprocessing}

1. Take input image

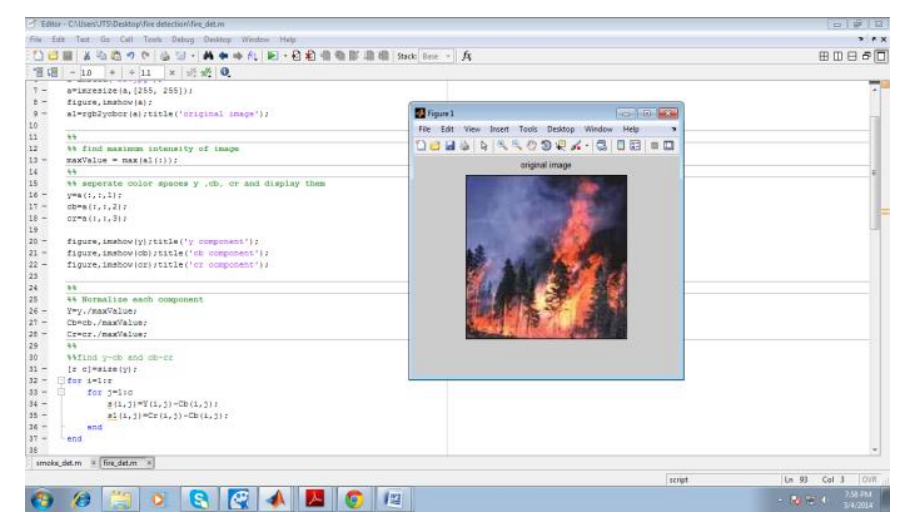

2. Convert into ycbcr color space.

3. Display each color space. 


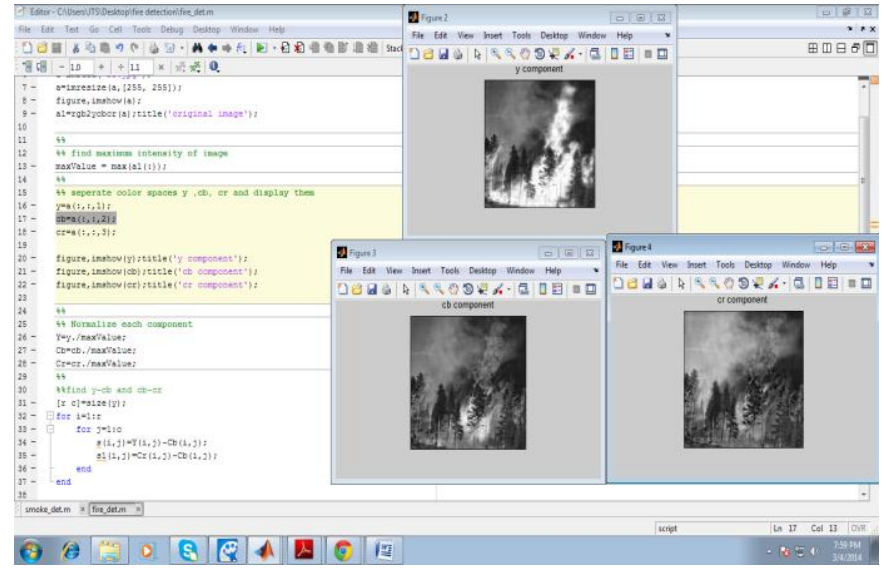

4. Normalize each color space component.

\subsubsection{Image Comparison using Wavelet}

Wavelet Based image Comparison

1. Read $1^{\text {st }}$ image

2. Resize it

3. Then decompose image using 2-D DWT

4. Read $2^{\text {nd }}$ image

5. Resize to same dimensions as $1^{\text {st }}$ image

6. Then decompose using 2-D DWT.

7. Compare sub bands of both images pixel by pixel.

8. If sub bands are equal then images are same else different.

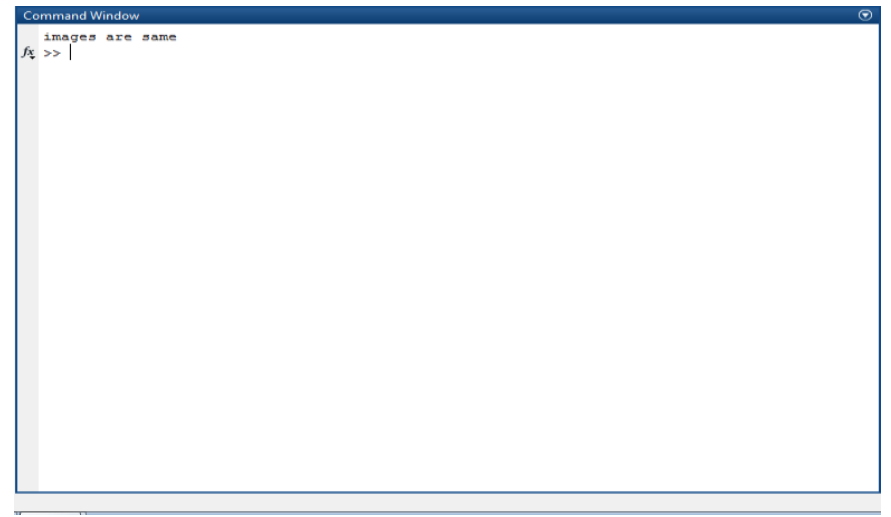

\section{Advantages Over Exist System}

Most of the algorithms do not take care of changing values of brightness due to change in weather conditions, and thus are not so practical for open area's, like forests, and hills etc. (Forest fires being a very big issue in United States).

No algorithm has tried to estimate the amount of smoke getting generated, so as to make an early warning system. If multiple camera's are synchronized together, then the camera's away from the source will register more smoke, but are not useful for ringing an alarm, as they donot have any information about the source of the fire.

\section{CONCLUSION}

The proposed system should be more robust in effectively discrimination of fire \& fire liked colored objects in audio \& video. The model should achieve high correct fire \& smoke detection rate with a low false alarm rate. As a future work, without any complicated rule system can be easily detect fire and smoke with the help of the proposed model.

\section{ACKNOWLEDGMENTS}

Thanks to my honourable Guide Ms. Varsha Bhosale, Lecturer in vidyalankar, Wadala for giving me valuable guidance.

\section{REFERENCES}

[1]. B. $U^{`}$ gur Toroyin $1, Y^{\sim}$ githan Dedeo glu2, and A. Enis $C$, etin 1 , "WAVELET BASED REAL-TIME SMOKE DETECTION IN VIDEO"

[2]. A. Proch'azka*, J. Pt'a a`cek*, and I. 'Sindel'a rov'a, "WAVELET TRANSFORM IN SIGNAL AND IMAGE RESTORATION"

[3]. Pao-Yen Lin, "An Introduction to Wavelet Transform" [4]. Surya.T.S, Suchithra.M.S, "Survey on Different Smoke Detection Techniques Using Image Processing".

[5]. Damir Krstinić, Darko Stipaničev, Toni Jakovčević, "HISTOGRAM-BASED SMOKE SEGMENTATION IN FOREST FIRE DETECTION SYSTEM".

[6]. www.google.com 